\title{
Raman Spectroscopic comparative study of Oxytocin and Freeze-dried Extract of Uvariodendron anisatum Verdeck (Annonaceae) and their influence on diet induced obesity in Sprague Dawley rats
}

Zephania Birech $^{1 *}$, Prabjot K. Sehmi ${ }^{2 * *}$, Peter W. Mwangi ${ }^{2}$, Nelly M. Nyaga ${ }^{2}$

${ }^{1}$ Department of Physics, University of Nairobi, 30197, Nairobi, Kenya

${ }^{2}$ Department of medical Physiology, University of Nairobi, 30197, Nairobi, Kenya

Corresponding authors: *birech@uonbi.ac.ke; ** prabjotsehmi@gmail.com

The authors have withdrawn this preprint because it was erroneously reported that oxytocin and UAV were administered orally. This was incorrect as both oxytocin and UAV extracts were intraperitoneally injected to the rats. A corrected version of the same manuscript is already published in preprints with identifier doi: https://doi.org/10.1101/554956 under title "Raman Spectroscopic Study of the Influence of Oxytocin and Uvariodendron anisatum Verdeck (Annonaceae) Freeze-dried Extracts on Diet Induced Obesity in Sprague Dawley Rats. 